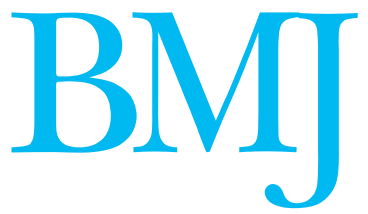

\title{
New Year's resolutions
}

\author{
Mind the gap between intention and behaviour
}

$\mathrm{N}$

ew Year's Day is nothing but an arbitrary day that, for instance, was celebrated by the Babylonians at the new moon after the spring equinox (and next year, the 1 Nisanu 2631 will be on what we call 9 April 2005 (www.cf-software.com/ other.html)). On 1 January no check, no pause, no planetary escapement in the celestial clockwork will take place as our planet spins relentlessly around its sun. Its only importance is for the human mind.

A complex history places New Year's Day just seven days after the great religious and commercial festival of Christmas. The week between Yule and Hogmanay is known in German as zwischen den Jahren (between the years), the magical period when little work gets done, next Christmas is a year away, and there is time for reflection. And since "to make an end is to make a beginning," as T S Eliot said, many new beginnings are resolved in those days. "Hell," though, according to $\mathrm{St}$ Bernard of Clairvaux, "is full of good intentions or desires," many no doubt being failed New Year's resolutions. Bridget Jones began her famous diary with 33 resolutions, and 364 days later wrote, "Number of New Year resolutions kept 1 (v.g.). An excellent year's progress."

"Must emigrate" will be a resolution of many young doctors in the United Kingdom, if one can extrapolate from a recent study in which $66 \%$ "did not definitely intend to practise medicine in the United Kingdom for the foreseeable future." ${ }^{2}$ However, as a commentator asked, ${ }^{3}$ do junior doctors actually do what they say? Longitudinal data show that many do not. ${ }^{4}$ When do intentions predict behaviours?

Some intentions definitely predict actions. My intention to write this editorial resulted in action, and I expect action from the editor's intention to pay me. Everyday life relies on intentions predicting actions: "I intend to cook dinner; I intend to collect the children from school." Some career intentions by doctors also predict actions, as in another study from the UK Medical Careers Research Group, 64\% of graduates intending to be psychiatrists were practising in psychiatry 10 years later, compared with $3 \%$ of those preferring other specialties. $^{5}$

However, experience tells us that even strong intentions need not result in action. Many patients intend to give up smoking, drink less, and exercise more, and yet no action occurs. Such gaps between intention and behaviour ${ }^{6}$ are the outcome of most New capacity for self deception, why do intentions sometimes not result in actions?

Sometimes intentions are not intentions, but are actually desires: "Arthur desires to leave medicine but does not intend to do so." "Freud emphasised that human behaviour is overdetermined, many factors motivating or discouraging even simple actions. We all have Walter Mitty fantasies, and some of the multitalented people who become doctors will inevitably speculate about alternative careers. Such needs, motivations, and desires may ultimately surface through a change of emphasis or direction within medicine.

Intentions can also be a cry for help, most obviously in a patient's stated intention to commit suicide. Questionnaire surveys, like protest votes at elections, allow a non-committal expression of discontent. Surveys on stress in doctors often find high apparent rates of psychiatric caseness, but formal psychiatric evaluation finds a low validity for such cases, ${ }^{8}$ as single issue questionnaires inflate apparent dissatisfaction and unhappiness.

Even the most solid of intentions can fail, as the fickle finger of fate causes "a slip 'twixt cup and lip." Some people, however, have more slips than others. People differ in their tendency to procrastination, ${ }^{9}$ and procrastination is particularly easy without a precise time window. How many doctors would have answered definitely if asked, "Are you intending to leave medicine in the next three months?" And how many would have signed a resignation letter dated three months hence? Intentions are anticipations of the future, and people differ in the perceived costs, benefits, and consequences of possible actions. Every action is another action denied, and the anticipated regret for lost possible futures may itself inhibit action.

Intentions are private mental events, and actions alone cannot tell us about them, particularly if the intention is to mislead about true intentions. Observers can also mislead themselves by wrongly inferring intentions from behaviours: "You never said you wanted to leave so we assumed you intended to continue practising as a doctor."

My intention is not to make any New Year's resolutions-interpret that as you will.

Chris McManus professor of psychology and medical education

University College London, Gower Street, London WC1E 6BT (i.mcmanus@ucl.ac.uk) 
Competing interests: CM intends to set up a biotech company for global marketing of an adenovirus vector for human transfection with the resolve gene of lemmings, which is upregulated by simultaneous serum uisge beatha and Wernicke area AuldLangSyne detection. Well, maybe, if he gets round to it.

1 Fielding H. Bridget Jones's diary: a novel. London: Picador, 1996.

2 Moss PJ, Lambert TW, Goldacre MJ, Lee P. Reasons for considering leaving UK medicine: questionnaire study of junior doctors' comments. $B M J$ 2004;329:1263-5.

3 Coebergh JA. More than words? [Electronic response to Moss et al]. BMJ 2004. bmj.bmjjournals.com/cgi/eletters/329/7477/1263.
4 Lambert TW, Goldacre MJ, Parkhouse J. Intentions of newly qualified doctors to practise in the United Kingdom. BMJ 1997:314:1591-2.

Goldacre MJ, Turner G, Fazel S, Lambert T. Career choices for psychiatry: Goldacre MJ, Turner G, Fazel S, Lambert T. Career choices for psychiatry:
national surveys of graduates of 1974-2000 from UK medical schools. $B r$ national surveys of graduates of 1974-2000 from UK medical schools. $\mathrm{Br}$
JPsychiatry (in press).

6 Sheeran P. Intention-behavior relations: a conceptual and empirical review. Eur Rev Soc Psychol 2002;12:1-36.

Perugini M, Bagozzi RP. The distinction between desires and intentions. Eur J Soc Psychol 2004;34:69-84.

8 Weinberg A, Creed F. Stress and psychiatric disorder in healthcare professionals and hospital staff. Lancet 1999;355:533-7.

9 Dewitte S, Schouwenburg HC. Procrastination, temptations, and incentives: the strugele between the present and the future in

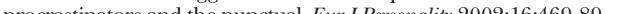

\title{
Out of body experiences and their neural basis
}

\author{
They are linked to multisensory and cognitive processing in the brain
}

"I

was in bed and about to fall asleep when I had the distinct impression that I was at the ceiling level looking down at my body in the bed. I was very startled and frightened; immediately (afterwards) I felt that I was consciously back in the (body on the) bed again." Out of body experiences, as described by a person here, are characterised by a location of the self (or one's centre of awareness) outside one's body, an impression of seeing the world from an extracorporeal elevated perspective, and an impression of seeing one's own body from this perspective. ${ }^{1-3}$ They are striking phenomena because they challenge the experienced spatial unity of self and body-or the experience of a real me that resides in one's body and is the subject of experience and action. ${ }^{45}$ Recent neurological evidence shows that these experiences are related to an interference with the temporo-parietal junction of the brain.

A better understanding of out of body experiences might further our scientific concepts about self and body and their experienced spatial unity. These have been influenced by diverse fields such as theology, philosophy, and psychology as well as neurology and psychiatry. ${ }^{1-7}$ Out of body experiences occur in about $10 \%$ of the population, most of the world's cultures, and several medical conditions. ${ }^{1-3}$ To date only few scientific investigations have been carried out on out of body experiences, probably because they generally occur spontaneously, are of short duration, and happen only once or twice in a lifetime. ${ }^{12}$ Investigations of neurological patients with out of body experiences are also rare, but they have several advantages. Out of body experiences in these patients might occur repetitively, sometimes in short succession, allowing for more detailed questioning of the experiences and associated sensations shortly after they occur. Moreover, researchers can analyse the associated neurological, causative, and anatomical findings.

Some clinicians have observed out of body experiences in association with various neurological conditions, but mainly in epileptic seizures and migraine. ${ }^{67}$ These early reports have also allowed us to link out of body experiences with deficient visual, vestibular, and multisensory processing. ${ }^{7}$ More recently, the importance of vestibular and multisensory mechanisms in out of body experiences was underlined by their occurrence in several patients with such experiences. ${ }^{3}$ In addition, vestibular illusions (of elevation, rotation, flying, lightness) and multisensory illusions (of visual limb shortening and movement) could be evoked by electrical stimulation of the same cortical area where higher stimulation currents induced out of body experiences. ${ }^{8}$ These data indicate that vestibular illusions, multisensory illusions of body parts (such as visual shortening and movement of limbs and phantom $\operatorname{limbs}^{9}$ ), and multisensory illusions of the entire body (such as out of body experiences) might share similar functional and anatomical mechanisms. ${ }^{3}$

Devinsky et al described several patients with out of body experiences caused by circumscribed brain damage and found that lesions predominantly affected the temporal lobe. ${ }^{6}$ More recently, our team analysed the lesions of several patients with out of body experiences and found that the temporo-parietal junction was affected in all patients. ${ }^{3}$ These patients had epilepsy and migraine. On the basis of these findings, our team proposed a cognitive model for out of body experiences, proposing that they are related to a failure of integration of proprioceptive, tactile, and visual information of one's body (personal space). ${ }^{3}$ This may lead to the experience of seeing one's body in a position (that is, on the bed) that does not coincide with the felt position of one's body (that is, under the ceiling). In this model disembodiment and elevated visuospatial perspective during out of body experiences are assumed to be related to additional vestibular dysfunction. ${ }^{3}$ In summary, the neurological evidence shows that out of body experiences are related to a disintegration within personal space (multisensory dysfunction) and a disintegration between personal space (vestibular) and extrapersonal space (visual) due to interference with the temporo-parietal junction.

In science the most challenging phenomena are often the ones we take for granted in our everyday lives. Excellent examples are the perception of the self and the experienced spatial unity between self and body. As argued by others, both folk psychological notions are challenged by out of body experiences. ${ }^{45}$ The reviewed evidence from neurological patients experiencing this striking dissociation between self and body shows that out of body experiences are culturally invariant phenomena that can be investigated scientifically.

The study of the self by neuroscience is in its infancy with no established models, very few data, and often not even the vocabulary to describe notions of 